\title{
ANTIMONIAIS EMPREGADOS NO TRATAMENTO DA LEISHMANIOSE: ESTADO DA ARTE
}

\author{
Susanne Rath*, Luciano Augusto Trivelin, Talitha Rebecca Imbrunito, Daniela Maria Tomazela, Marcelo Nunes de Jesús \\ e Percy Calvo Marzal \\ Instituto de Química, Universidade Estadual de Campinas, CP 6154, 13084-971 Campinas - SP \\ Heitor Franco de Andrade Junior e André Gustavo Tempone \\ Instituto de Medicina Tropical de São Paulo, Universidade de São Paulo, Av. Dr. Enéas de Carvalho Aguiar, 470, 05403-000 \\ São Paulo - SP
}

Recebido em 27/5/02; aceito em 15/1/03

\begin{abstract}
ANTIMONIALS EMPLOYED IN THE TREATMENT OF LEISHMANIAISIS: THE STATE OF THE ART. Antimony preparations are the drugs of choice for the treatment of leishmaniasis over 90 years, a disease that currently affects 12 million people worldwide. Its introduction was based on $19^{\text {th }}$ century concepts of therapeutic effects of metal salts as arsenicals and other metals, most of them abandoned due to toxic effects or better drugs. In the last three decades, there was a great improvement in the knowledge of cell biology and immunology of those infections, but chemotherapy has not been improved in the same strength. The structure and mechanism of action of the two pentavalent antimonial drugs of choice, meglumine antimoniate and sodium stibogluconate, are not well known and the contamination of those pharmaceutical by toxic contaminants have been verified.
\end{abstract}

Keywords: antimony; leishmaniasis; therapy.

\section{INTRODUÇÃO}

\section{Leishmaniose}

A leishmaniose é uma doença infecciosa zoonótica, amplamente distribuída em todo mundo, que afeta o homem e os animais. Esta parasitose ocorre na Ásia, Europa, África e Américas, sendo que existem relatos sobre a doença, no continente americano, desde a época colonial. Em 1571, Pedro Pizarro relatou que os povos situados nos vales quentes do Peru eram dizimados por uma doença que desfigurava o nariz, a qual foi posteriormente caracterizada como leishmaniose ${ }^{1}$. A importância desta doença era tamanha, que as deformações provocadas ${ }^{2}$ chegaram a ser registradas em peças cerâmicas por artistas da época.

A descoberta dos agentes etiológicos das leishmanioses, entretanto, só ocorreu o final do século XIX, quando Cunningham em 1885, na Índia, descreveu formas amastigotas em casos de Calazar. Em 1898, o pesquisador russo Borovisky demonstrou ser um protozoário o agente etiológico do Botão do Oriente. Em 1903, Leishman e Donovan realizaram as primeiras descrições do protozoário responsável pelo Calazar indiano, denominado mais tarde de Leishmania donovani. Igualmente em 1903, Wright descreveu o parasita do Botão do Oriente, conhecido atualmente como Leishmania tropica ${ }^{3}$.

A partir de 1904, diferentes relatos demonstram que o calazar não ocorria exclusivamente na Índia, visto que alguns casos também foram registrados na China.

Como o Calazar na região do Mediterrâneo atingia principalmente crianças, as evidências de diferenças entre o organismo causador do Calazar de uma região para outra justificaram o estabelecimento de uma espécie Leishmania infantum por Nicole, em 1908. Um organismo semelhante foi descrito por Wright em $1903 \mathrm{em}$ uma criança com Botão do Oriente na Síria, sendo proposto o nome de

*e-mail: raths@iqm.unicamp.br
Helcosoma tropicum para este parasita. Mais tarde ele foi colocado no gênero Leishmania: Leishmania tropica, Wright 1903.

No continente americano, várias doenças que criavam lesões, freqüentes em determinadas regiões, eram chamadas de úlcera de Bauru, ferida brava, uta, úlcera dechiclero. A correlação destas lesões com um protozoário do gênero Leishmania foi estabelecida por Gaspar Vianna, em 1909, no Instituto Oswaldo Cruz, recebendo o nome de Leishmania braziliensis.

Em 1993, a Organização Mundial da Saúde considerou a Leishmaniose como a segunda doença de importância pública, causada por protozoário.

\section{VETORES}

Os vetores da leishmaniose são dípteros da família Psychodida, hematófagos pertencentes aos gêneros Phlebotomus (Velho Mundo) e Lutzomyia (Novo Mundo), com vasta distribuição nos climas quentes e temperados. Somente as fêmeas são hematófagas. Pertencem ao tipo dos dípteros de atividade crepuscular e pós-crepuscular, abrigando-se durante o dia em lugares úmidos, sombrios e bem protegidos dos ventos. São encontrados em tocas de animais silvestres, buracos de pau, ocos de bambu ${ }^{4}$.

Os flebótomos, dos gêneros Lutzomyia e Psychopopygus, infectam-se ao picar o animal portador da doença, aspirando macrófagos parasitados ou amastigotas livres no sangue ou tecidos e podem, assim, transmitir a doença ao homem ${ }^{5}$. Os mamíferos portadores da leishmaniose são geralmente animais silvestres como a preguiça, o tamanduá, roedores, raposas e outros, sendo que grande parte das lesões nestes não é aparente. No Brasil, o mais importante reservatório animal é o cão e a raposa ${ }^{6}$. A partir de estudos patofisiológicos foi verificado que a Leishmania se desenvolve no tubo intestinal do hospedeiro invertebrado, na forma promastigota, e essa, uma vez introduzida nos mamíferos através da picada, transforma-se na forma amastigota. Enquanto a forma promastigota é flagelada e extracelular, a forma amastigota é intracelular e sem movimento. A multiplicação dos amastigotas ocorre no interior de vacúolos parasi- 
tóforos em macrófago de diferentes tecidos, originando a doença na forma cutânea, mucocutânea e visceral.

No Novo Mundo, são reconhecidas oito espécies de Leishmanias responsáveis pela doença no homem, pertencentes ao subgênero Vianna (V) e Leishmania (L), onde os agentes etiológiocos correspondentes são: Leishmania (V) braziliensis, Leishmania (V) guyanensis, Leishmania (V) panamensis, Leishmania (V) lainsoni, Leishmania (L) mexicana, Leishmania (L) amazonensis, Leishmania (L) venezuelensis e Leishmania (L) chagasi ${ }^{7}$.

Basicamente podemos diferenciar duas formas de leishmaniose: a Leishmaniose Tegumentar Americana (LTA) e a Leishmaniose Visceral Americana (LVA).

No Estado de São Paulo, o agente etiológico responsável pela ocorrência da doença, principalmente da forma cutânea, é a Leishmania (V) braziliensis.

\section{ÁREAS ENDÊMICAS}

A leishmaniose prevalece nos quatro continentes, sendo considerada endêmica em 88 países, dos quais 72 são países considerados em desenvolvimento.

Segundo a Organização Mundial da Saúde 90\% dos casos de leishmaniose visceral são registrados em Bangladesh, Brasil, Nepal, Índia e Sudão; 90\% dos casos da leishmaniose mucocutânea ocorrem no Brasil, Bolívia e Peru e 90\% dos casos da leishmaniose cutânea ocorrem no Afeganistão, Brasil, Irã, Peru, Arábia Saudita e Síria ${ }^{8}$.

No Brasil, a leishmaniose visceral encontra-se disseminada em 17 estados das regiões Nordeste, Centro-Oeste e Sudeste. A ampliação da área de distribuição geográfica da AIDS e sua extensão para camadas mais pobres da população e, por outro lado, a crescente urbanização da leishmaniose visceral faz supor que a prevalência da co-infecção LVA / HIV venha a aumentar nos próximos anos. Entre 1985 e 2000, a leishmaniose atingiu no Brasil 422,5 mil pessoas e nos últimos dois anos foram detectados 66,8 mil novos casos da doença, que permanece sem controle ${ }^{9}$. De 1999 a 2001, foram registrados no Estado de São Paulo, 87 casos de LVA, sendo que em sete casos a doença levou a óbito ${ }^{10}$.

\section{DIAGNÓSTICO DA DOENÇA}

De modo geral o diagnóstico da doença é baseado no exame clínico dos sintomas e histórico fornecidos pelo paciente. Um dos principais problemas quanto a esse diagnóstico inicial é a semelhança do quadro clínico da leishmaniose visceral com algumas doenças linfoproliferativas e com a esquistossomose mansônica associada à bacteriose septicêmica prolongada. O diagnóstico laboratorial pode ser realizado por (i) ensaios sorológicos, entre os quais destaca-se o ensaio imunoadsorvente ligado à enzima (ELISA) e o de imunofluorescência indireta (IFI) e (ii) exame parasitológico, realizado no material colhido por punção na medula óssea, baço ou fígado, onde o material é examinado em lâminas coradas, inoculado em cultura ou em hamster ou cultivado em meios apropriados. Ainda podem servir para diagnosticar a doença a análise do hemograma e dosagem de proteínas. Dependendo da forma clínica, pode ocorrer uma diminuição do número total de hemáceas, leucopenia com linfocitose relativa e plaquetopenia, bem como inversão da relação albumina/ globulina ${ }^{11}$.

\section{FÁRMACOS EMPREGADOS NO TRATAMENTO DA LEISHMANIOSE}

Apesar do uso medicinal de compostos de antimônio já ser conhecido desde a Antigüidade, séculos antes da era cristã, para diversos fins terapêuticos, somente em 1912, Gaspar de Oliveira Vianna ${ }^{12}$ observou que o tártaro emético era eficaz na terapêutica da leishmaniose tegumentar americana. Três anos mais tarde, na Itália, também foi comprovada a eficácia desta droga no tratamento de Calazar ${ }^{13}$.

Devido aos efeitos tóxicos e graves efeitos colaterais indesejáveis associados ao emprego do tártaro emético, e.g. intolerância gastrintestinal e efeitos cardiotóxicos, os antimoniais trivalentes (Tabela 1) foram sendo substituídos por compostos estibiados pentavalentes (Tabela 2). Bramachari, em 1920, desenvolveu o primeiro composto à base de antimônio pentavalente, o uréia estibamina, derivado uréico do ácido p-aminofenil estibínico. Em 1936, Schmidt introduziu na terapia médica o gluconato de antimônio (V) sódico, conhecido comercialmente como Solustibosan ${ }^{\circledR}$ (Bayer) ou Pentostam $^{\circledR}$ (Glaxo Wellcome $)^{5,14}$.

Tabela 1. Estrutura química de antimoniais trivalentes empregados na clínica médica, com os respectivos nomes químicos e comerciais

$$
\begin{aligned}
& \text { Fórmula estrutural } \\
& \left\{\begin{array}{c}
\mathrm{O} \\
\| \\
\mathrm{CO}- \\
I \\
\mathrm{HO}-\mathrm{Sb}-\mathrm{OH} \\
\mathrm{HCO}- \\
\mathrm{COOH}
\end{array}\right\} \mathrm{K}
\end{aligned}
$$$$
\text { Nome químico/ }
$$$$
\text { Nome comercial }
$$

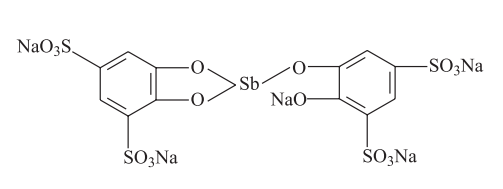

Antimoniato de

bis-catecol-3,5-

dissulfonato sódico ${ }^{16}$

Stibophen, Repodral,

Fuadina

$$
\stackrel{\mathrm{O}}{\mathrm{I}}=\stackrel{\mathrm{C}}{\mathrm{C}}-\mathrm{O}-\mathrm{Sb}-\mathrm{S}-\mathrm{CH}_{2} \mathrm{COONa}
$$

Tioglicolato de sódio e antimônio ${ }^{16}$

Durante a Segunda Guerra Mundial, surgiu na França um medicamento alternativo ao até então gluconato de antimônio(V) sódico, o antimoniato de N-metil glucamina, comercializado como Glucantime ${ }^{\circledast}$ (Rhône-Poulenc-Rohrer) ou antimoniato de meglumina. Enquanto o Pentostam ${ }^{\circledR}$ é distribuído, até hoje, nos países de língua inglesa, o Glucantime ${ }^{\circledR}$ manufaturado é comercializado nos países de línguas francesa e espanhola.

Um estudo comparativo ente o medicamento original e o genérico correspondente do gluconato de sódio antimônio, realizado no Quênia, revela a viabilidade de comercialização do medicamento a custos menores e, em consequiência, a possibilidade do tratamento de maior número de pacientes infectados pela leishmaniose visceral na África ${ }^{15}$.

\section{ANTIMONIATO DE METILGLUCAMINA}

No Brasil, o medicamento à base de antimônio, utilizado como primeira escolha na terapêutica da leishmaniose, é o antimoniato de metilglucamina. O composto é obtido sinteticamente a partir do ácido antimônico e da N-metil-glucamina, sendo a última obtida previamente a partir da aminação redutora da glicose em presença de metila- 
Tabela 2. Estrutura química de antimoniais pentavalentes empregados na clínica médica

\begin{tabular}{ll}
\hline Fórmula estrutural proposta & Nome químico \\
& Nome comercial
\end{tabular}

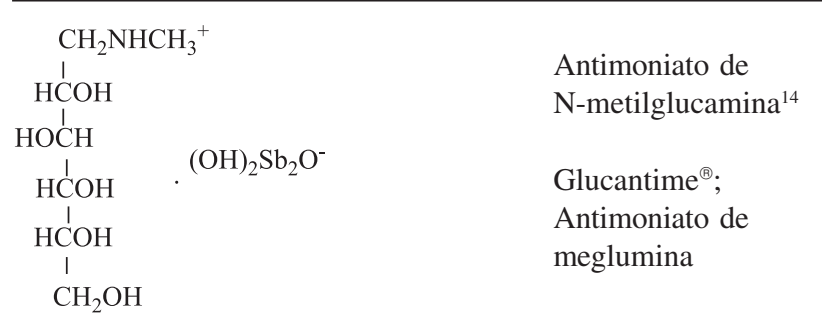

\begin{tabular}{|c|c|c|}
\hline 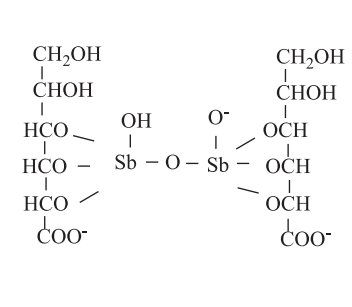 & $\mathrm{Na}_{3} .9 \mathrm{H}_{2} \mathrm{O}$ & $\begin{array}{l}\text { Gluconato de } \\
\text { antimônio }(\mathrm{V}) \\
\text { sódico }{ }^{14,17} \\
\text { ou Estibogluconato } \\
\text { de sódio } \\
\text { Pentostam } \\
{ }^{\circledR} \\
\text { Solustibosan }^{\circledR}\end{array}$ \\
\hline
\end{tabular}

Estibamine ${ }^{\circledR}$

mina. O composto, de fórmula estrutural não definida, é solúvel em água e pouco solúvel em solventes orgânicos.

$\mathrm{O}$ antimoniato de metilglucamina é especialmente eficaz no tratamento de leishmaniose cutânea, mucocutânea e visceral. O medicamento provoca regressão rápida das manifestações clínicas e hematológicas da doença, bem como provoca a esterilização do parasita.

Devido às baixas dosagens e tratamentos descontínuos, começaram a ocorrer falhas na terapia e conseqüente aumento das formas resistentes de parasitas ${ }^{18-20}$. A Organização Mundial de Saúde preconiza que as doses de antimoniais não devem ultrapassar $20 \mathrm{mg} / \mathrm{kg} /$ dia, não se ultrapassando o limite de $850 \mathrm{mg}$ de antimônio ${ }^{18}$, devido à sua elevada toxicidade. Mialgias, dores abdominais, alterações hepáticas e distúrbios cardiológicos são efeitos colaterais freqüientemente associados ao uso destas drogas ${ }^{18}$.

Após administração endovenosa ou intramuscular, o antimoniato de metilglucamina é rapidamente absorvido e, praticamente, $90 \%$ do antimônio é excretado nas primeiras $48 \mathrm{~h}$ pelos rins ${ }^{16}$. Em conseqüência, faz-se necessária a administração de doses elevadas do fármaco, em regime contínuo, para garantir um elevado teor de antimônio nos tecidos e, assim, obter a eficácia do tratamento. Efeitos colaterais como nefrites, distúrbios gastrintestinais, cardiovasculares e respiratórios têm sido observados. Antimoniais pentavalentes são geralmente 10 vezes menos tóxicos em células de mamíferos, quando comparados aos antimoniais trivalentes (tártaro emético), utilizados primeiramente por Vianna em 1912 $2^{21}$. Em determinados casos, além de destruir o parasita, o medicamento acaba por levar o paciente ao óbito ${ }^{14}$. $\mathrm{O}$ antimônio ainda pode ser detectado no cabelo do paciente tratado com antimoniais pentavalentes após um ano do término do tratamento ${ }^{22}$.
Apesar do emprego do antimoniato de metilglucamina no tratamento da leishmaniose por mais de 50 anos, a estrutura e composição do composto, igualmente ao gluconato de antimônio (V) sódico, ainda permanecem indeterminadas.

Os dados mais recentes sobre a tentativa de caracterização do antimoniato de metilglucamina foram reportados por Roberts et al. ${ }^{21}$. Segundo os autores, a razão molar antimônio: N-metil-D-glucamina é de 1:1,37 e várias espécies complexas entre o antimônio e N-metilD-glucamina coexistem em solução. Oligômeros foram observados, sendo a maior massa molar encontrada de 507 uma. Espécies de forma geral (NMG-SB)n-NMG, onde NMG: N-metil-D-glucamina, foram identificadas. Demicheli et al. ${ }^{23}$ sintetizaram o antimoniato de metilglucamina e sugeriram que o antimônio se liga na metilglucamina através do oxigênio do carbono C-3.

No entanto, a eficácia terapêutica destas formas poliméricas ou mesmo do antimônio pentavalente ainda não foi esclarecida. Há indícios que o $\mathrm{Sb}$ (III) é substancialmente mais potente do que o $\mathrm{Sb}(\mathrm{V})$ contra promastigotas e amastigotas de, pelo menos, três espécies de Leishmania. Esses resultados reforçam a hipótese de uma conversão metabólica intramacrofágica do $\mathrm{Sb}(\mathrm{V})$ em $\mathrm{Sb}(\mathrm{III})$ sendo, neste caso, o $\mathrm{Sb}(\mathrm{III})$ o elemento tóxico às leishmanias no estado intracelular ${ }^{19}$. Pouco se compreende ainda sobre o mecanismo de ação desta droga e, sugere-se que o antimônio pentavalente possa ser uma pró-droga, sendo convertido a antimônio trivalente após sua admistração ${ }^{21}$, e que assim possa interferir no processo de $\beta$-oxidação de ácidos graxos e glicólise do parasita, levando à uma depleção dos níveis de ATP intracelular ${ }^{18}$. Além disso, existe na forma amastigota uma metaloprotease zinco dependente, que poderia ser inativada se o antimônio substituísse o zinco nesta enzima, essencial para o desenvolvimento do parasita ${ }^{24}$. Recentemente, Miekeley et al. ${ }^{25}$ verificaram que, após administração intramuscular de antimoniato de N-metilglucamina em pacientes com leishmaniose, ocorre conversão in vivo do composto orgânico estibiado para as formas iônicas $\mathrm{Sb}^{3+} \mathrm{e} \mathrm{Sb}^{5+}$. Ainda, foi observado que ocorre a bio-redução do $\mathrm{Sb}^{5+}$ para a sua forma trivalente, corroborando outros estudos que evidenciam que a formação in vivo do $\mathrm{Sb}^{3+}$ seja responsável tanto pela toxicidade da droga como pela atividade terapêutica da mesma.

\section{OUTROS AGENTES QUIMIOTERÁPICOS UTILIZADOS NO TRATAMENTO DA LEISHMANIOSE}

Além dos antimoniais, outras drogas têm sido empregadas no tratamento das diversas formas da leishmaniose, entre as quais se destacam a pentamidina, anfotericina $B$, paromomicina e o miltefosine (Tabela 3).

A descoberta da atividade quimioterápica das substâncias do grupo das diamidas, do qual faz parte a pentamidina, foi inteiramente fortuita. A pentamidina, além de ser relativamente eficaz na terapia da leishmaniose, é eficaz no tratamento de casos incipientes de tripanossomíase gambiense ou rodesiana ${ }^{26}$. A pentamidina é comercializada sob o nome de Lomidina ${ }^{\circledR}$ e encontra-se disponível, nos Estados Unidos, somente no Serviço de Medicamentos para Doenças Parasitárias do Serviço de Saúde Pública. No tratamento da leishmaniose visceral (leishmaniose por L. donovani, ou Calazar) a pentamidina foi usada com sucesso, em séries de 12 a 15 doses. A segunda série, administrada após intervalo de 1 a 2 semanas, pode ser necessária em áreas onde se sabe que a infecção responde de modo insatisfatório ao tratamento. A substância é particularmente útil em casos que não responderam aos antimoniais ou para pacientes com Calazar que sejam hipersensíveis ao antimônio. A alta toxicidade desta droga também é fator limitante para o uso. Hipoglicemia, hipotensão, alterações cardiológicas, nefrotoxicidade e, até mesmo, morte repentina foram descritas ${ }^{18}$. 
Tabela 3. Outras drogas empregadas na terapia da leishmaniose, com os respectivos nomes químicos e comercial

\begin{tabular}{ll}
\hline Fórmula estrutural & $\begin{array}{l}\text { Nome Químico/ } \\
\text { Nome comercial }\end{array}$ \\
\hline
\end{tabular}

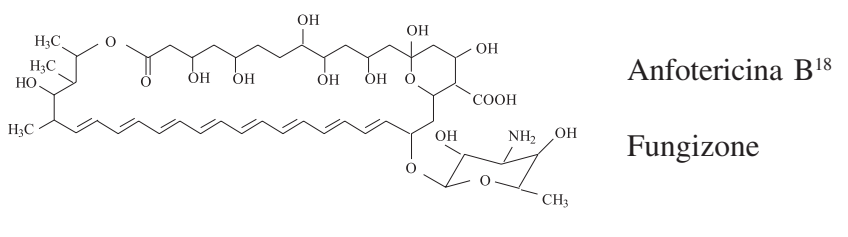

$\mathrm{O}$
II
$\mathrm{CH}_{3}-\left(\mathrm{CH}_{2}\right)_{14}-\mathrm{CH}_{2}$-O-P-O- $\left(\mathrm{CH}_{2}\right)_{2}-\mathrm{N}^{+}\left(\mathrm{CH}_{3}\right)_{3} \quad$ Miltefosine
O

Humatin

A anfotericina B é um antibiótico antifúngico derivado de uma cepa de Streptomyces nodosus, sendo indicada para o tratamento da leishmaniose mucocutânea americana, embora não se considere fármaco de primeira eleição. A anfotericina B pode ser incorporada em lipossomas carregadores sendo absorvida pelo sistema reticuloendotelial onde o parasita da leishmania reside, e é assim pouco absorvido pelos rins, o maior órgão alvo para a toxicidade da anfotericina B. Estudos de fase II, em pequena escala, determinaram a dose mínima efetiva e os resultados confirmaram que o AmBisome ${ }^{\mathrm{TM}}$ é consideravelmente menos tóxico que a anfotericina $\mathrm{B}$ convencional. O AmBisome ${ }^{\mathrm{TM}}$ provou ser eficaz tanto no tratamento da leishmaniose cutânea como mucocutânea, sendo utilizado nos casos onde existe falha com a terapia de antimoniais ${ }^{27}$. Sua administração mais eficaz dá-se por via endovenosa e como as outras, também possui nefrotoxicidade e pode levar à depleção dos níveis de potássio e magnésio no organismo ${ }^{28}$.

Outro medicamento que se tem mostrado efetivo contra a leishmaniose visceral é a paromomicina (também chamada aminosidine), antibiótico aminoglicosídeo que é ativo contra espécies de leishmania in vitro e in vivo. Estudos clínicos, para se testar a eficácia da paromomicina injetável contra a leishmaniose visceral, têm sido realizados na Índia, onde o tratamento antimonial padrão não é muito efetivo e as taxas de mortalidade são altas. Outro medicamento ainda utilizado no arsenal terapêutico é o alopurinol, vantajoso especialmente por sua administração oral e baixa toxicidade, porém há diversos casos relatados em que esta droga não foi eficaz no controle da infecção ${ }^{29}$.

Atualmente, todos os medicamentos disponíveis para o tratamento da leishmaniose são injetáveis. Foi verificado que o miltefosine, uma droga anti-câncer alquilfosfolipídica, é ativo contra Leishmania spp, in vitro e in vivo, e pode vir a ser o primeiro tratamento oral para a leishmaniose visceral. Resultados de estudos de fase II, na Índia, indicam que quando o miltefosine é oralmente administrado é bem tolerado. Em todas as doses testadas, a droga produziu excelentes resultados de cura parasitológica (contra Leishmania donovani). Estudos clínicos de fase III do miltefosine contra leishmaniose visceral estão sendo planejados ${ }^{30}$.

\section{TOXICOLOGIA DO ANTIMÔNIO}

Antimônio é considerado um agente clastogênico, mas não mutagênico no seu estado trivalente, sendo esta espécie responsável pelos principais efeitos tóxicos. Ainda não foi comprovada a carcinogenicidade do antimônio em humanos, embora o $\mathrm{Sb}_{2} \mathrm{O}_{3}$ seja classificado, pelo IARC, como possível carcinógeno humano ${ }^{31}$. Estudos epidemiológicos ocupacionais não comprovam para humanos, a ocorrência de câncer de pulmão causada pelo antimônio em animais de experimentação, particularmente ratos fêmeas ${ }^{32-34}$. As poucas informações existentes sobre os aspectos toxicológicos do antimônio são provenientes de estudos de compostos orgânicos à base de antimônio de uso farmacológico ${ }^{35}$.

$\mathrm{O}$ antimônio acumula-se, em geral, em órgãos vascularizados e tecidos, principalmente rins e fígado, além de possuir grande afinidade pelo baço e pelo sangue. Uma quantidade expressiva de antimônio no sangue pode estar relacionada com a grande afinidade do $\mathrm{Sb}$ (III) pelos eritrócitos ${ }^{36}$.

O metabolismo do antimônio está relacionado com a mudança de valência após incorporação no organismo vivo, aproximadamente de $5-10 \%$. No homem, assim como em roedores, apenas uma pequena quantidade de $\mathrm{Sb}(\mathrm{V})$ é reduzida para a forma trivalente. Entretanto, quantidades similares de Sb (III) são oxidadas para o estado pentavalente. A evidência de metilação de antimônio em mamíferos é baixa. Assim sendo, parece que o organismo não possui mecanismos eficientes de detoxificação do $\mathrm{Sb}(\mathrm{III})^{37}$.

A excreção de $\mathrm{Sb}$ (III) e (V) em humanos é preferencialmente via renal. Mais que $80 \%$ do $\mathrm{Sb}(\mathrm{V})$ administrado intravenosamente como estibogluconato é excretado na urina após 6-8 $\mathrm{h}^{38}$. A cinética, de compostos de antimônio pentavalente (estibogluconato e antimonato de meglumina), após injeção intramuscular, pode ser descrita por um modelo de dois compartimentos e três termos farmacocinéticos, representando uma fase inicial de absorção com tempo de meia vida de $0,85 \mathrm{~h}$, seguida de uma fase de eliminação rápida com tempo de meia vida de 2,02 h e, por último, uma fase de eliminação com tempo médio de meia vida de $76 \mathrm{~h}^{37,39}$.

\section{METODOLOGIAS ANALÍTICAS PARA A DETERMINAÇÃO DE ANTIMÔNIO}

Diversas metodologias analíticas têm sido desenvolvidas para a determinação de antimônio em matrizes como sedimento, material biológico, ligas metálicas, medicamentos, alimentos, bebidas e outros. No entanto, pelo fato do antimônio ser um elemento tóxico cumulativo, que foi detectado em águas naturais, a matriz água tem sido a mais estudada. A especiação do antimônio nas diferentes matrizes é fundamental, visto que a toxicidade e o comportamento biológico são dependentes do estado de oxidação.

Muitos métodos permitem apenas a determinação do elemento em um dos estados de oxidação, sendo o outro determinado indiretamente, por diferença entre o antimônio total e o previamente determinado. Neste sentido, as técnicas hifenadas são promissoras, visto que permitem simultaneamente separar e quantificar as espécies $\mathrm{Sb}(\mathrm{III})$, $\mathrm{Sb}(\mathrm{V})$ e organoantimoniais, diminuindo assim a introdução de erros provenientes da determinação indireta. As técnicas hifenadas representam uma tendência atual no desenvolvimento de metodologias analíticas, entre as quais se destacam, entre outras, a eletroforese capilar com espectrometria de massas com fonte de plasma acoplado induti- 
vamente (CE-ICP-MS), cromatografia líquida de alta eficiência com espectrometria de fluorescência atômica com geração de hidretos (HPLC-HG-AFS) e a cromatografia líquida de alta eficiência com espectrometria de absorção atômica e geração de hidretos (HPLC-HGAAS $)^{40}$. Embora esses métodos sejam indubitavelmente promissores para a especiação de antimônio, ainda são pouco viáveis para serem empregados no controle e qualidade de medicamentos, onde se requer métodos simples, baratos e de elevada frequiência analítica.

Metodologias analíticas reportadas na literatura para a determinação de antimônio em fármacos recomendam, principalmente, métodos volumétricos e/ou potenciométricos. Os métodos volumétricos são baseados na redução do $\mathrm{Sb}(\mathrm{V})$ para $\mathrm{Sb}(\mathrm{III})$ com um agente redutor, geralmente iodeto em meio ácido. O iodo formado no processo é evaporado e $\mathrm{Sb}$ (III) determinado por iodometria. Essa metodologia não é adequada, visto que permite apenas a determinação de antimônio total. Ainda, está sujeita a erros decorrentes de uma eliminação incompleta do iodo formado anteriormente à determinação do $\mathrm{Sb}(\mathrm{IIII})$.

A farmacopéia brasileira ainda não apresenta monografia para o doseamento de antimônio no antimoniato de metilglucamina. A determinação de antimônio pentavalente no estibogluconato de sódio, segundo a monografia contida na Farmacopéia Britânica, é realizada mediante titulação potenciométrica do antimônio com sulfato ferroso amoniacal. No entanto, essa metodologia, além de ser lenta, carece de precisão. Bloomfield et al. ${ }^{41}$ descreveram um método por injeção em fluxo para a determinação de antimônio pentavalente no Pentostam. No entanto, até então, nenhuma das metodologias acima citadas faz referência a determinação seletiva de $\mathrm{Sb}$ (III) e (V) nos fármacos estudados.

A importância da especiação do antimônio no fármaco é importante, visto que o efeito terapêutico versus toxicidade está intimamente relacionado com o estado de oxidação do elemento. Nesse sentido, é importante que as monografias apresentadas nas farmacopéias sejam revistas. A contaminação do antimoniato de metilglucamina por antimônio (III) foi relatada por Franco et al..$^{42}$ e Rath et $a l .{ }^{43}$ empregando diversas metodologias como a polarografia de pulso diferencial, espectrometria de absorção atômica, espectrometria de emissão ótica com plasma indutivamente acoplado e a espectrofotometria.

Cabe destacar, que a Agência Nacional de Vigilância Sanitária do Ministério da Saúde do Brasil colocou, recentemente, em estudo e consulta pública uma monografia para o antimoniato de meglumina a ser introduzida no $4^{\circ}$ Fascículo da Farmacopéia Brasileira. Nesta monografia, sugere-se o doseamento do antimônio trivalente e pentavalente por espectrometria de absorção atômica com geração de hidretos em batelada, com atomização em cela de quartzo, sendo que o $\mathrm{Sb}(\mathrm{V})$ é previamente reduzido por uma solução redutora de tetraidroborato de sódio em meio alcalino ${ }^{44}$.

\section{CONCLUSÕES}

A leishmaniose é uma doença infecciosa que vem aumentando consideravelmente em todo mundo. Nas últimas três décadas, houve um grande avanço no conhecimento da biologia celular e imunologia nestas infecções, mas a quimioterapia não foi aprimorada em igual amplitude. Preparações de antimônio são as drogas de escolha no tratamento das leishmanioses, nos últimos 90 anos. Sua introdução foi baseada em conceitos terapêuticos do século XIX, onde era significativa a participação terapêutica de sais de metais, como arsênio e outros.

A indústria pouco tem contribuído no desenvolvimento de novos medicamentos para o tratamento da leishmaniose e, no Brasil, a droga de eleição continua sendo o antimoniato de metilglucamina. A estrutura e composição desta droga ainda não foram totalmente esclarecidas, e o mecanismo de ação ainda é pouco conhecido. Existem fortes evidências que o antimônio pentavalente seja reduzido in vivo à sua forma trivalente, o que vem a explicar a toxicidade da droga, bem como seu efeito terapêutico. Ainda, o medicamento disponível no Brasil tem apresentado problemas quanto à sua qualidade.

Quanto às metodologias analíticas, ainda existe uma deficiência quanto à disponibilidade de métodos simples e de baixo custo que permitam a especiação de antimônio e que possam ser implantados em laboratórios de controle de qualidade de medicamentos, visando não somente a determinação do teor de $\mathrm{Sb}(\mathrm{V})$, mas também de possíveis contaminantes tóxicos.

\section{AGRADECIMENTOS}

Os autores agradecem à FAPESP e ao CNPq por auxílios e bolsas concedidas. Em homenagem ao Médico, Cientista e Professor P. Marsden, in memorian, que tanto contribuiu para os avanços na terapia da leishmaniose no Brasil.

\section{REFERÊNCIAS}

1. Goldman, L.; Arch. Dermatol. 1983, 119, 540

2. http://www.dbbm.fiocruz.br/tropical/leishman/indice.html, acessada em Setembro 2002.

3. Hoare, C. A.; Trans. R. Soc. Trop. Med. Hyg. 1938, 32, 67.

4. http://www.sucen.sp.gov.br/doencas/index.htm, acessada em Maio 2002.

5. Berman, J. D.; Rev. Infect. Dis. 1988, 10, 560.

6. http://www.funasa.gov.br/pub/GVE/GVE0517A.htm, acessada em Maio 2002.

7. Grimaldi, G.; Tesh, A. B.; McMahon, P. D.; Am. J. Trop. Med. Hyg. 1989, $41,689$.

8. http://www.who.int/emc/diseases/leish/leisgeo1.html, acessada em Maio 2002.

9. Zorzetto, R.; Pesquisa FAPESP 2001, 68, 53.

10. http://www.cve.saude.sp.gov.br/htm/leishvis.htm, acessada em Maio 2002.

11. http://www.cve.saude.sp.gov.br/htm/inf_lva2000.htm, acessada em Maio 2002.

12. Vianna, G. O.; Anais do $7^{\circ}$ Congresso Brasileiro de Medicina e Cirurgia 1912, 4, 426.

13. Di Christina, G.; Caronia, G.; Bull. Soc. Pathol. Exot. 1915, 8, 63.

14. Marsden, P. D.; Rev. Soc. Bras. Med. Trop. 1985, 18, 187.

15. Moore, E.; O'Flaherty, D.; Heuvelmans, H.; Seaman, J.; Veeken, H.; Wit, S.; Davidson, R. N.; Bulletin of the World Health Organization 2001, 79, 388 .

16. Limongi, J. P. Em Farmacodinâmica; Corbett, C. E., ed.; Livraria Editora Artes Médicas: São Paulo, 1973, cap. 61.

17. Goldsmith, R. S. Em Farmacologia Básica e Clínica; Katzung, B. G., ed.; Guanabara Koogan S.A.: Rio de Janeiro, 1998, cap. 54.

18. Balaña-Fouce, R.; Reguera, R. M.; Cubría, C.; Ordóñez, D.; Gen. Pharmacol. 1998, 30, 435.

19. Sereno, D.; Lemesre, J. L.; Antimicrob. Agents Chemother. 1997, 41, 972.

20. Berman, J. D.; Wyler, D. J.; J. Inf. Diseases 1980, 142, 83.

21. Roberts, W. L.; McMurray, W. J.; Rainey, P. M.; Antimicrob. Agents Chemother. 1998, 42, 1076.

22. Dórea, J. G.; Costa, J. M. L.; Holzbecher, J.; Ryan, D. E.; Marsden, P. D.; Clin. Chem. 1987, 33, 2081.

23. Demicheli, C.; Figueiredo, T. L.; Carvalho, S.; Sinesterra, R. D.; Lopes, J. C. D.; Frezard, F.; BioMetals 1999, 12, 63.

24. Bangs, J. D.; Ransom, D. A.; Nimick, M.; Christie, G. H.; Mol. Biochem. Parasitol. 2001, 114, 111.

25. Miekeley, N.; Mortari, S. R.; Schubach, A. O.; Anal. Bioanal. Chem. 2002, $372,495$.

26. Doua, F.; Miezan, T. W.; Singaro, J. R. S.; Yapo, F. B.; Baltz, T.; Am. J. Trop. Med. Hyg. 1996, 55, 586.

27. Yardley, V.; Croft, S. L.; Int. J. Antimicrob. Agents 2000, 13, 243.

28. Rang, H. P.; Dale, M. M. Em Pharmacology; $2^{\text {nd }}$ ed., Churchill Livingstone: UK, 1991.

29. Martinez, S.; Marr, J. J.; New Engl. J. Med. 1992, 326, 741.

30. Fischer, C.; Voss, A.; Engel, J.; Medical Microbiol. Immunolog. 2001, 190, 85.

31. http://www-cie.iarc.fr/htdocs/monographs/vol47/47-11.htm, acessada em Setembro 2002. 
32. Gerhardsson, L.; Brune, D.; Nordberg, G. F.; Wester, P. O.; Scand. J. Work Environ. Health 1982, 8, 201.

33. Jones, R. D.; Occup. Environ. Med. 1994, 51, 772.

34. Groth, D. H.; Stettler, L. E.; Burg, J. R.; Busey, W. M.; Grant, G. C.; Wong, L.; J. Toxicol. Environ. Health 1986, 18, 607.

35. Gebel, T.; Chem.-Biol. Interact. 1997, 107, 131.

36. Felicetti, S. A.; Thomas, R. G.; McClellan, R. O.; Am. Ind. Hyg. Assoc. J. 1974, 35, 292.

37. Chulay, J. D.; Fleckenstein, L.; Smith, D. H.; Trans. R. Soc. Trop. Med. Hyg. 1988, 82, 69.

38. Rees, P. H.; Kesting, M. I.; Kager, P. A.; Hockmeyer, W. T.; Lancet 1980, 2,226 .
39. Pamplin, C. L.; Desjadins, R.; Chulay, J.; Tramont, E.; Henricks, L.; Canfield, C.; Clin. Pharm. Ther. 1981, 29, 270.

40. Krachler, M.; Emons, H.; J. Anal. At. Spectrom. 2001, 16, 20.

41. Bloomfield, M. S.; Dow, D. A.; Prebble, K. A.; J. Pharm. Biomed. Anal. 1992, 10, 779 .

42. Franco, A.; Barbosa, A. C.; Rath, S.; Dórea, J. G.; Am. J. Trop. Med. Hyg. 1995, 52, 435.

43. Rath, S.; Jardim, W. F.; Dórea, J. G.; Fresenius' J. Anal. Chem. 1997, 358, 548.

44. http://www.ufsm.br/farmacopeia/.html, acessada em Setembro 2002. 